\title{
Innovation Opportunities in Critical Results Communication: Theoretical Concepts
}

\author{
Bruce I. Reiner
}

Published online: 18 June 2013

(C) Society for Imaging Informatics in Medicine 2013

\section{Introduction}

Failure in communication between health care personnel has been reported to account for over $60 \%$ of root causes of sentinel events reported to the Joint Commission on Accreditation of Healthcare Organizations [1] and has prompted repeated calls from the Institute of Medicine for redesigning and error proofing health care delivery in order to improve communication and patient safety $[2,3]$. In radiology, communication failures are particularly common [4-6] and are a leading cause of medical malpractice [7-9] as well as dissatisfaction among referring physicians [10]. With so much negativity surrounding conventional radiology reporting and communication practice, it is a wonder as to why innovation in practice and technology has been lacking for so long.

The American College of Radiology has defined three categories of radiology critical results including emergent, discrepant, and unexpected findings, and requires the interpreting physician (i.e., radiologist) to expedite report delivery in a manner that ensures timely receipt of the findings [11]. The method of communication is largely left to the discretion of the radiologist, as long as "timely receipt" is ensured and documented. In the absence of specific and standardized communication standards, institutions and practitioners are often left to their own device, which can lead to differing expectations and practices on the parts of interpreting radiologists and referring clinicians. In the event that an adverse clinical outcome does result and communication is determined to be a contributing factor, contradictory claims are often made by involved radiologists and clinicians, as to the method, content, and timeliness of communication. In the absence of objective and reproducible data, establishing causality of the communication

B. I. Reiner $(\bowtie)$

Department of Radiology, Veterans Affairs Maryland Healthcare System, 10 North Greene Street, Baltimore, MD 21201, USA

e-mail: breiner1@comcast.net error is often impossible and this leads to the potential for repeating past mistakes. The ultimate goal is the creation of standardized communication databases which longitudinally record, track, and analyze all critical results communications and supporting data while simultaneously creating a method of qualitative and quantitative accountability.

\section{Current Practice: Challenges and Limitations}

At the same time, health care providers are subjected to increasing data overload; service requirements are consistently increasing with providers asked to provide timelier service. From a radiology reporting standpoint, report turnaround times are now being measured in hours (or even minutes) rather than days, with the adoption of speech recognition technology and teleradiology. While these technologic and practice advances result in radiology report data being readily and almost instantaneously accessible after exam completion, the reality is that long periods of time often transpire before the data are reviewed and acted upon by referring clinicians. With the abundance of multidisciplinary data consumed by a physician in the course of everyday practice, it is not unusual for a physician to delay or even overlook data from a given radiology report. In these circumstances, the limiting factor is not the radiology report "turnaround time" but instead the radiology report "action time." This radiology report action time can be defined from the time of radiology report completion to the time clinical action takes place based upon the report findings. While action time delays for routine or expected report findings may not necessarily be time urgent, delays associated with critical results may often be associated with increased morbidity and/or mortality. This has led to the creation of critical results reporting requirements, with the stated goal of ensuring that critical findings contained within the radiology report are communicated and acted upon in a timely fashion. 
But given the current state of data overload (and data fatigue), are existing critical results communication strategies accomplishing their stated goals?

The answer to this question in large part varies in accordance with the specific nature of the critical finding, institutional standards, workload and state of mind of the involved parties (i.e., interpreting radiologist and referring clinician), technology in use, method of communication employed, and degree of accountability. In many circumstances, the involved parties are too busy, frustrated, or preoccupied to personally engage in communication. As a result, the radiologist or clinician may often delegate communication responsibilities to third parties (e.g., nurse, clerical staff, and technologist) who often lack detailed knowledge and/or understanding of the findings being communicated. At the same time, the method of communication used by the radiologist may often consist of a hand written note, which has the potential to be misread, leading to error. The end result is that the communication may have taken place in theory, but the resulting clinical actions lacked in timeliness and/or accuracy. In the proverbial sense, the operation was a success, but the outcome was a failure.

While attempts have been made to mandate direct physician to physician communication, they are often hard to enforce, due to time and availability constraints. Physicians are often unwilling to wait for prolonged periods on the telephone and as a result either defer to colleagues or computers. With the advent of computers throughout medical practice, attempts have been made to utilize computermediated communication (CMC) as a means to facilitate prompt and targeted task-oriented interactions. In addition, CMC offers an additional advantage of eliminating the requirement that critical results be read back by the health care worker when communication takes place by telephone [12]. In theory, CMC addresses many of these methodological and outsourcing concerns, and also provides the added benefit of data documentation. In practice however CMC is often unsuccessful due to the lack of receipt acknowledgment and follow through on the part of the referring clinician $[4,13$, 14]. Data overload is believed to be a principle factor accounting for these failures, with an excessive number of computerized alerts regularly encountered [15-17].

The end result is that existing communication strategies often fall short of their intended goals, and this perpetuates the potential for communication errors and deficient clinical outcomes. New strategies are required which can overcome existing deficiencies but not overload already stressed human resources.

\section{Innovation Opportunity}

There are a number of different stakeholder perspectives to consider when defining an innovation opportunity. In medical

imaging practice, the primary "clinical provider" perspectives are those of the radiologist (i.e., imaging service provider) and clinician (i.e., clinical service provider). While these "clinical provider" perspectives are of the highest importance in effecting clinical outcomes, additional perspectives for consideration include those of the patient, administrator, payer, and technology provider. While an innovation providing practical gain to the clinical provider has true value, an innovation which provides gain to multiple perspectives has the potential to be truly transformative and this should be the theoretical goal for any proposed innovation.

Innovation from the clinical provider perspective is primarily focused on data, which lies at the core of all medical decision making. If we are contemplating the creation of innovation in critical results communication, we must therefore focus on the data of greatest importance (Table 1). In order to effect timely and accurate decision making, the radiologist should be able to communicate to the referring clinician the finding and/or diagnosis of concern, the type of critical result (i.e., classification), its anatomic location, degree of urgency, and follow-up recommendations. In order to minimize the potential for misunderstanding and expedite clinical action, the mode of communication should be direct, concise, devoid of ambiguity, verifiable, and readily reviewable. While verbal communication has traditionally been used for critical results communication in medicine, it often lacks many of these requirements and has been cited as a frequent source of error [18] while not being easily tracked and analyzable. An alternative strategy using electronic communication could in theory address many of these requirements but has the perceptual disadvantage of depersonalizing communication. The ideal solution would be to create an innovation strategy which combines the two, through the creation of a standardized electronic communication tool, along with multidirectional consultation capabilities (which can integrate gestures, annotations, and speech as consultation input options).

The goals and objectives of the proposed critical results communication innovation are listed in Table 2 and consist of three fundamental requirements which are aimed at maximizing workflow and operational efficiency, data standardization and analysis, and accountability to ensure compliance and optimized clinical outcomes. In order to optimize end-user acceptance, we should attempt to make the proposed innovation workflow neutral (i.e., new workflow equal to or superior to its predecessor), incorporate

Table 1 Recommended data requirements for critical results communication
1. Classification

2. Follow-up recommendations

3. Anatomic location

4. Finding/diagnosis

5. Degree of urgency 
Table 2 Goals and objectives for critical results communication
A. Operational efficiency and workflow
1. Ease in use
2. Timeliness
3. Portability
4. Adaptability
5. Automation
B. Data: dynamic and documentable
1. Consistency
2. Understanding
3. Image centric
4. Accuracy
5. Longitudinal analytics
C. Accountability
1. Individual and institutional compliance
2. Standards and guidelines
3. Automated alerts and prompts
4. User authentication and identification
5. Hierarchical triggers and escalation pathways

automation whenever available, and create adaptive and customizable features which are tied to the end-user profile and individual preferences. The specific manner in which these features are incorporated into the proposed innovation and a representation of everyday use is discussed in detail in a companion article [19].

Since the data lie at the core of the proposed innovation, the specifics related to the type of data, methods of input and presentation, and resulting analytics are essential to the clinical efficacy and perceived value. In order to create a consistent methodology for outcomes analysis, it is essential that data be recorded in a standardized fashion, which in turn can lead to the creation of a referenceable database, which is not a practical option for most existing models of critical results communication due to use of non-standardized written free text or spoken methods of communication. The challenge is to create a workflow efficient method for capturing and recording standardized data without limiting perceived expressiveness, which has been cited as a deficiency of existing structured reporting systems [20]. The principle strategy used to address these requirements is the use of selected medical images which best illustrate the critical results and then annotating the images using a standardized annotation and image markup schema (i.e., annotated key images), which has been proposed in other medical imaging applications [21]. The annotated images would in turn be supplemented by standardized textual data which provides complementary data relating to the specific finding, diagnosis/differential diagnosis, anatomic location, critical results classification, urgency, and follow-up recommendations. A representative standardized schema for reporting these data is listed in
Table 3. In order to improve workflow, end-users can create customizable finding-specific reporting macros, which can directly incorporate these standardized data and reduce or eliminate manual data input requirements. Examples of such finding-specific macros for a pneumothorax could include the following:

1. Small pneumothorax macro:

Size (10\%)

Anatomic location: Lung (right)

Classification: Unexpected

Urgency: Subacute

Follow-up recommendation: Chest radiograph in 4-6 hours

2. Moderate pneumothorax macro:

Size $(25 \%)$

Anatomic location: Lung (right)

Classification: Emergent

Urgency: Subacute

Follow-up recommendation: Surgical intervention (thoracostomy tube)

3. Tension pneumothorax macro:

Size $(70 \%)$

Anatomic location: Lung (right)

Classification: Emergent

Urgency: Hyperacute

Follow-up recommendation: Surgical intervention (thoracostomy tube)

In these examples, one can see that the same finding (i.e., pneumothorax) can be differentiated into three separate macros based upon size, urgency, and recommended followup. Other than requiring input as to the specific size and laterality, all additional data elements are embedded in the
Table 3 Goals and objectives for critical results communication 
preformed macro, providing efficient workflow and data standardization.

In the event an end-user desired to expand the communication beyond that of the standardized data elements described, he/she would have the option to input free text or speech (i.e., using a voice file) into the key image data set for added expressiveness. While it would be saved as ancillary (i.e., unstructured) data and available for future review, it would not readily lend itself to longitudinal data analysis. The recording of standardized image, annotation, and text data provides a method for improving data consistency, conciseness, and understanding while also providing a methodology for clinical data mining. The addition of these standardized finding-specific annotated key images also provides a rich image-centric database for education and research.

Accountability is arguably one of the greatest deficiencies of existing critical results communication strategies and is an integral component of the proposed innovation strategy. The internal critical results communication requirements can be established and modified in accordance with national, institutional, departmental, or individual end-user guidelines. Automated triggers and alerts can be used to notify the individual end-user when an established criterion for critical results communication has been recognized (e.g., through natural language processing of contemporaneous radiology reports) and automatically open the critical results application. In the event the end-user elects to manually override the automated prompt, the denial and accompanying data could be recorded in a database for compliance and quality assurance review. Once the proposed critical results communication application has been engaged, the system could be modified to ensure that all mandatory data requirements are completed before the critical results communication is sent. If for example, a radiologist chose not to input data related to follow-up recommendation for an emergent finding (e.g., acute appendicitis), the system may automatically prompt the radiologist and prevent transmission until the requisite data requirements have been fulfilled.

The establishment and modification of critical results communication requirements is an ongoing and dynamic process. As changes are made at national or local levels, these can be directly integrated into the critical results application, to ensure compliance with new and/or revised standards and guidelines. It is not unusual for individual end-users to have different critical results communication requirements from one another (which go beyond the institutional or national norms), and these can be automatically programmed into the reporting and information system technology. As an example, one primary care physician may request critical results communication for all brain imaging studies performed to evaluate a potential stroke, regardless of observed findings. In this scenario, the system could be configured to automatically notify the radiologist and open up the critical results communication application at the time of interpretation.
While the primary onus of critical results communication in radiology has largely been placed on the interpreting radiologist, many communication failures are the result of lack of clinician availability or follow-up [4, 22]. Current critical results communication models often rely on manual communication attempts to ensure that the critical results are successfully communicated, with frequent outsourcing of responsibilities to third parties. Once the communication has been successfully achieved, the customary practice is to document the date, time, and names involved in the communication as an addendum in the radiology report, with little or no follow-up as to whether ensuing clinical actions took place in an accurate and/or timely fashion. This relative lack of accountability in current practice provides an excellent opportunity for innovation, while simultaneously providing longitudinal analysis of context and user accountability. In addition to obvious analysis of end-user responsiveness and follow through, the data could also be used to identify context-specific system-wide deficiencies (e.g., operating room availability and surgical completion times for acute appendicitis in the emergency room). The ability to prospectively track and analyze these data provides the theoretical advantage of identifying deficiencies and intervening before they become so severe as to result in a major adverse event (e.g., mortality). If for example a physician on call is frequently delayed in responding to emergent critical results communications, the department chief would be notified once the trend was established and place heightened scrutiny and monitoring on physician responsiveness. One method may be to modify the escalation pathway for this particular physician on call, so that instead of waiting the customary 20 minutes for notification of the second tier physician, the system may be modified to wait only 10 minutes before escalating to the next level.

A number of existing technologies can be integrated into the proposed critical results communication strategy to enhance quality performance, security and data analysis. These technologies could include natural language processing software (for report data mining to identify critical results requiring communication), biometrics (for end-user authentication and identification), and electronic tracking tools such as RFID (to track provider location and availability). The integration of biometrics would not only provide enhanced data security but also provide unequivocal documentation as to the identity of the person receiving a critical results communication. At the same time, if an end-user's location is outside of the communications network or the network is non-functioning, the system would automatically trigger an escalation pathway and divert communications to the second tier party. The purpose is not intended to be punitive but instead to be proactive to insure that critical results communications are completed in an accurate and timely fashion. These safeguards are currently lacking in conventional 
practice and could serve as a step in the right direction, with the hopes of reducing errors in communication and effecting improved health care outcome

\section{References}

1. Arora V, Johnson J, Lovinger D, et al: Communication failures in patient sign-out and suggestions for improvement: a critical incident analysis. Qual Saf Health Care 14:401-407, 2005

2. Kohn LT, Corrigan JM, Donaldson MS (eds.): To Err Is Human: Building a Safer Health System. Vol 627. Washington, DC: National Academies, 2000.

3. Aspden P, Corrigan JM, Wolcott J, Erickson SM (eds.): Patient Safety: Achieving a New Standard of Care. Washington, DC: National Academies, 2004.

4. Singh H, Arora HS, Vij MS, et al: Communication outcomes of critical imaging results in a computerized notification system. J Am Med Inform Assoc 14:456-460, 2007

5. Roy CL, Poon EG, Karson AS, et al: Patient safety concerns arising from test results that return after hospital discharge. Ann Intern Med 143:121-128, 2005

6. Anthony SG, Prevedello LM, Damiano MM, et al: Impact of a 4 year quality improvement initiative to improve communication of critical imaging test skills. Radiology 259:802-807, 2011

7. Berlin L: Statute of limitations and the continuum of care doctrine. AJR 177:1011-1016, 2001

8. Berlin L: Communicating findings of radiologic examinations: whither goest the radiologists' duty? AJR 178:809-815, 2002

9. Johnson CD, Krecke KN, Miranda R, et al: Quality initiatives: developing a radiology quality and safety program - a primer. RadioGraphics 29:051-959, 2009
10. Poon EG, Gandhi TK, Sequist TD, et al: "I wish I had seen this test result earlier!" dissatisfaction with test result management systems in primary care. Arch Intern Med 164:2223-2228, 2004

11. Kushner DC, Lucey LL: Diagnostic radiology report and communication: the ACR guideline. J Am Coll Radiol 2:15-21, 2005

12. Haverstick DM: Critical value called, read-back obtained. Am J Clin Pathol 121:790-791, 2004

13. Ash JS, Berg M, Coiera E: Some unintended consequences of information technology in healthcare: the nature of patient care information system-related errors. J Am Med Inform Assoc 11:104-112, 2004

14. Wahls TL, Cram PM: The frequency of missed test results and associated treatment delays in a highly computerized health system. BMC Fam Prac 8:32, 2007

15. Taylor LK, Tamblyn R: Reasons for physicians non-adherence to electronic drug alerts. Stud Health Technol Inform 107:1101-1105, 2004

16. Van der Sijs H, Aarts J, Vulto A, et al: Overriding of drug safety alerts in computerized physician order entry. J Am Med Inform Assoc 13:138-147, 2006

17. Feldstein A, Simon SR, Schneider J, et al: How to design computerized alerts to safe prescribing practices. Jt Comm J Qual Saf 30:602-613, 2004

18. Sutcliffe KM, Lewton E, Rosenthal MM: Communication failures: an insidious contributor of medical mishaps. Acad Med 79:186194, 2004

19. Reiner B. Innovation opportunities in critical results communication: practical solutions. J Digit Imaging 2013 (in press).

20. Langlotz CP: Structured radiology reporting: are we there yet? Radiology 253:23-25, 2009

21. Channin DS, Mongkolwat P, Kleper V, et al: The caBig annotation and image markup project. J Digit Imaging 23:217-225, 2010

22. Gandhi TK, Sittig DF, Franklin M, et al: Communication breakdowns in the outpatient referral process. J Gen Intern Med 15:626631,2000 